\title{
Universal formula for the muon-induced neutron yield
}

\author{
N. Yu. Agafonova* and A. S. Malgin \\ Institute For Nuclear Research of the Russian Academy of Sciences \\ 60th October Anniversary Prospect 7a, 117312 Moscow, Russia
}

(Dated: September 7, 2018)

\begin{abstract}
The experimental data on the yield of muon-induced neutrons for liquid scintillator, iron and lead accumulated during 60 years of muon interaction underground study have been analyzed. A universal formula connecting the yield with muon energy loss in the matter and neutron production in hadronic and electromagnetic showers is presented.
\end{abstract}

PACS numbers: 25.30.Mr

Keywords: neutron yield, muons

Intriduction.-In the last decade a renewed interest to the problem of yield $Y_{n}$ of muon-induced neutrons becomes evident. This is due to both the increased requirements to the accuracy of background definition in underground experiments and the growth of computing resources. The yield dependence on both the mean muon energy $\bar{E}_{\mu}$ and atomic weight $A$ of the medium has been investigating using the FLUKA and GEANT simulation packages and their versions [1], 2], [3], [4]. The yield calculation results are significantly different (Fig.1 in [2]). The normalization of calculations to the experimental data becomes more complicated due to large straggling in these data. Until now no expression exists for $Y_{n}$ that binds together the muon energy deposition, nuclear properties of the matter and the neutron production processes in hadronic (h) and electromagnetic (em) showers generated by muons and developing in the matter. To calculate a yield value the approximate empirical laws $Y_{n}=p_{E} \times E_{\mu}^{\alpha}$ (for fixed $A$ ) or $Y_{n}=p_{A} \times A^{\beta}$ (for fixed $\left.E_{\mu}\right)$ are used. The constants $\alpha, \beta$ are defined based on the results of calculations. Numerical fitting coefficients $p_{E}, p_{A}$ are entered to get the agreement between calculations and a set of experimental data available.

The form of dependence $Y_{n}\left(\bar{E}_{\mu}\right)=a \bar{E}_{\mu}^{\alpha}$ was proposed in 5. As follows from results of the measurements [6], [7], 8], 9] and calculations [1], [2], [5], 10], [1], the $\alpha$ value is in the range from 0.7 to 0.9 . The values of exponents $\alpha$ and $\beta$ representing the contributions of the neutron production channels play an important role in the analysis of the experimental and calculated data.

Experimentally, the yield $Y_{n}$ is given by

$$
Y_{n}=\frac{N_{n}}{\bar{l}_{\mu} \rho}\left(\mathrm{n} / \mathrm{g} / \mathrm{cm}^{2}\right),
$$

where $N_{n}$ is the number of neutrons produced by a muon at energy $\bar{E}_{\mu}$ on the path length $\bar{l}_{\mu}$ in the matter with density $\rho . \bar{E}_{\mu}$ and $\bar{l}_{\mu}$ are mean values for muon flux at a depth $H$ and muon track lengths, respectively. The yield is connected with the medium properties and the characteristics of reactions of neutron production by expression:

$$
Y_{n}=\frac{N_{0}\langle\nu \sigma\rangle}{A},
$$

where $N_{0}$ is the Avogadro number, $\langle\nu \sigma\rangle$ is a mean value of the product of photonuclear $\mu A$-interaction cross section and neutron multiplicity $\nu, A$ is the atomic weight of the medium. Equation (2) follows from the dependence $N_{n}$ on $\langle\nu \sigma\rangle$ and $\bar{l}_{\mu}$

$$
N_{n}=c_{A}\langle\nu \sigma\rangle l_{\mu}=\frac{\rho N_{0}}{A}\langle\nu \sigma\rangle=\frac{\langle\nu \sigma\rangle}{A} \rho l_{\mu} N_{0},
$$

where $c_{A}\left[\mathrm{~cm}^{-3}\right]$ is a concentration of nuclei $A$.

Experimental data.-The table lists the measured yield $Y_{L S}$ for liquid scintillator (LS), $\mathrm{Fe}\left(Y_{F e}\right), \mathrm{Pb}\left(Y_{P b}\right)$. The table covers almost all the data obtained for about 60 year measurements of the neutron yield in underground experiments. The data are listed in the order of increasing of energy $\bar{E}_{\mu}$ to which authors attributed their result. An error in determining average muon energy $\bar{E}_{\mu}$ was only shown in Ref. [12]. To estimate the $\bar{E}_{\mu}$ value error in other experiments summarized in the table we have used the expression $\delta \bar{E}_{\mu}=2 / \sqrt{\bar{E}_{\mu}}$. It covers both the uncertainty $\bar{E}_{\mu}$ calculations at different sets of parameters offered in Ref. [13], 14], [15] and deviations of $\bar{E}_{\mu}$ values from the $\bar{E}_{\mu}(H)$ dependence which can be seen in the table. The Mote Carlo calculations carried out recently [16], [17] have resulted in a revision some $Y_{L S}$ values. The most part of the LS data [6], [8], [11], [18], [19], 20], 21] was obtained using scintillator $C_{n} H_{2 n}$ $n=9.6 \rho=0.78 \mathrm{~g} / \mathrm{cm}^{3}$ [6], 11], 20], 21] (in the table the refined value $Y_{L S}=4.1 \times 10^{-4} \mathrm{n} / \mu /\left(\mathrm{g} / \mathrm{cm}^{2}\right)$ from 22] is included).

It should be noted that the paper [3] cites wrongly (Table IV in [3]) the results of Ref. [6], [8], 20], namely, out of 15 values taken from these works and included in the Table IV, seven values do not correspond to the published original data. The correct values of $H, \bar{E}_{\mu}$ and $Y_{L S}$ are presented in the table of given paper and also in Ref. [4]. 
TABLE I: Measured neutron yield

\begin{tabular}{|c|c|c|c|c|c|c|}
\hline \multirow[b]{2}{*}{$\bar{E}_{\mu}, G e V$} & \multicolumn{4}{|c|}{$Y_{n} \times 10^{-4}, n / \mu /\left(\mathrm{g} / \mathrm{cm}^{2}\right)$} & \multirow[b]{2}{*}{ Ref. } & \multirow[b]{2}{*}{ year } \\
\hline & H, m.w.e. & $Y_{L S}$ & $Y_{F e}$ & $Y_{P b}$ & & \\
\hline$\overline{10.0 \pm 6.3^{a}}$ & 20 & - & $0.98 \pm 0.01$ & $2.43 \pm 0.13$ & {$[26]$} & 1954 \\
\hline $10.0 \pm 6.3^{a}$ & 60 & - & - & $4.8 \pm 0.6$ & [27] & 1970 \\
\hline $11.0 \pm 6.6^{a}$ & 40 & - & $1.32 \pm 0.30$ & $4.03 \pm 0.36$ & [9] & 1971 \\
\hline $13.0 \pm 7.2$ & 20 & $0.20 \pm 0.07$ & - & - & [18] & 1995 \\
\hline $16.5 \pm 8.1$ & 32 & $0.36 \pm 0.03$ & - & - & [19] & 2000 \\
\hline $16.7 \pm 8.2$ & 25 & $\begin{array}{l}0.47 \pm 0.05 \\
0.36 \pm 0.05^{b}\end{array}$ & - & - & [6] & 1973 \\
\hline $17.8 \pm 8.4^{a}$ & 80 & - & $1.69 \pm 0.30$ & $5.66 \pm 0.36$ & [9] & 1971 \\
\hline $20 \pm 9^{a}$ & 110 & - & - & $6.8 \pm 0.9$ & [27] & 1970 \\
\hline $40 \pm 12.6^{a}$ & 150 & - & $3.31 \pm 0.96$ & $11.56 \pm 1.1$ & [28] & 1968 \\
\hline $86 \pm 18$ & 316 & $\begin{array}{l}1.21 \pm 0.12 \\
0.93 \pm 0.12^{b}\end{array}$ & - & - & [6] & 1973 \\
\hline $110 \pm 21^{a}$ & 800 & - & - & $17.5 \pm 3.0$ & [29] & 1970 \\
\hline $125 \pm 22$ & 570 & $\begin{array}{l}2.04 \pm 0.24 \\
1.57 \pm 0.24^{b}\end{array}$ & - & - & [20] & 1986 \\
\hline $260 \pm 8$ & 2700 & $2.8 \pm 0.3$ & - & - & {$[12]$} & 2010 \\
\hline $280 \pm 33$ & 4300 & - & - & $116 \pm 44$ & [30] & 1973 \\
\hline $280 \pm 33$ & 3100 & $\begin{array}{l}4.1 \pm 0.5 \\
3.3 \pm 0.5^{b}\end{array}$ & $16.4 \pm 2.3$ & - & [21] & 2005 \\
\hline $280 \pm 33$ & 3100 & $3.2 \pm 0.2$ & $19.0 \pm 1.0$ & - & [11] & 2011 \\
\hline $385 \pm 39$ & 5200 & $\begin{array}{l}5.3_{-1.02}^{+0.95} \\
4.1 \pm 0.6^{b}\end{array}$ & $20.3 \pm 2.6$ & - & [8] & 1989 \\
\hline
\end{tabular}

${ }^{a}$ Vertical flux

${ }^{b}$ Corrected values

The measurements were carried out in a global muon flux at different depths and energies $\bar{E}_{\mu}$ from $16.7 \mathrm{GeV}$ to $385 \mathrm{GeV}$. The experiments [6], [20] detected the neutrons produced only in the counter LS; the results of Ref. [8], [11], 21] covered the neutrons generated in LS and iron of the setup structures (LS and iron masses were almost equal).

The counters were located close to the mine ceiling of gypsum $\left(\bar{E}_{\mu}=16.7 \mathrm{GeV}\right)$ or salt $\left(\bar{E}_{\mu}=86 \mathrm{GeV}\right)$ in the experiment [6] and close to the mine ceiling of salt $\left(\bar{E}_{\mu}\right.$ $=125 \mathrm{GeV}$ ) in Ref. 20]. As a result of the Monte Carlo calculations in Ref. [16], it was obtained that the contribution of neutrons produced by shower particles in the standard rock around the detecting volume LS $\left(\mathrm{C}_{12} \mathrm{H}_{26}\right)$ enlarges the measured yield $Y_{L S}$ by $\sim 30 \%$. Taking into account this fact and disregarding the small difference between compositions of LS and rock in experiments [6], [20] and calculations [16], we have obtained the corrected values of $Y_{L S}$ which are presented in the table.

The similar correction is not suited for the results of [8], 21], 22] since in these experiments inner counters are detecting the neutrons produced in an inner volume of setup consisting of LS and iron in the same proportion as the peripheral part of the setup.

With LSD and LVD which are almost identical in design and detection technique the yield was measured under different conditions of neutron detection: a) with inner counters crossed by a muon (LSD [8]), b) with all counters of the inner setup volume crossed by a muon (LVD 21], 22]), c) with inner counters fired by any trigger pulse, including the muon trigger (LVD [23]). Here a muon is meant both a single muon and a muon group with shower accompaniment or without it. In all papers using the LSD and LVD data the yield was defined by formula

$$
Y_{L S}=\frac{N^{\text {det }}}{N_{\mu} \rho_{L S} \bar{l}_{L S} \eta},
$$

where $N^{\text {det }}=N_{L S}^{\text {det }}+N_{F e}^{\text {det }}$ is the number of detected neutrons, including produced in $\operatorname{LS}\left(N_{L S}\right)$ and iron $\left(N_{F e}\right)$, while $N^{\text {det }}=N_{L S} \eta_{L S}+N_{F e} \eta_{F e}$, where $\eta_{L S}, \eta_{F e}$ are corresponding neutron detection efficiencies; $Q$ is the fraction of neutrons produced in LS, $N_{\mu}$ is an amount of muons, $\bar{l}_{L S}$ is a mean length of muon tracks in LS. $N^{\text {det }}, N_{\mu}$ and $\bar{l}_{L S}$ have been determined directly in the experiment. $N_{\mu}$ has been determined with due regard to the multiplicity of muon groups established using the tracking system data [21]. The $\mathrm{Q}$ fraction was calculated 
with the assumption that $\eta=\eta_{L S}=\eta_{F e}$. The values of $Q, 0.61,0.60,0.85$ and $\eta=0.60,0.90,0.60$ have been used for cases a), b) c), respectively. Case c) leads to the selection of neutrons at energies above $10 \mathrm{MeV}$ and, as a consequence, to significant reduction in $Y_{L S}$ what was indicated in [21], 24]. For this reason, the result of [23] is not included in the table.

The recent Monte Carlo calculations in Ref. [17] showed that $\eta_{L S} \neq \eta_{F e}$. This leads to the need to change the formula (4):

$$
Y_{L S}=\frac{N^{\text {det }}}{N_{\mu} \rho_{L S} \bar{l}_{L S}} \times \frac{Q}{Q \eta_{L S}+(1-Q) \eta_{F e}} .
$$

Given fraction $Q$ the yield $Y_{F e}$ can be also defined

$$
Y_{F e}=\frac{N^{d e t}}{N_{\mu} \rho_{F e} \bar{l}_{F e}} \times \frac{1-Q}{Q \eta_{L S}+(1-Q) \eta_{F e}},
$$

where $\bar{l}_{F e}$ is a mean length of muon tracks in iron and $\rho_{F e}$ is the iron density.

New $Q$ values were calculated in 25] for cases a), b) of the neutron detection with LSD and LVD. The $Q$ fraction depends on the ratios of masses $k_{M}=M_{L S} / M_{F e}$, surface areas $k_{S}=S_{F e} / S_{L S}$ calculated per counter, atomic weights $k_{A}=A_{L S} / A_{F e}$, and the exponent $\beta$

$$
Q=\frac{k_{A}^{\beta} k_{M} k_{S}}{\left(1+k_{A}^{\beta} k_{M} k_{S}\right)} .
$$

On the basis of the LSD experimental data in 25] it was found that $\beta=0.95$ and $Y_{F e}=20.3 \times 10^{-4}$. To do this, the data of inner counters of first LSD level were used. In addition, these counters were detecting the neutrons produced by muons in a 8 -cm thick steel platform beneath the setup. Using the data of the inner LSD counters of the second level, the fraction $Q=0.138$ has been determined for case a). The $Y_{L S}$ value of $(4.1 \pm 0.6) \times 10^{-4}$ corresponds to this fraction at efficiencies $\eta_{L S}=0.45, \eta_{F e}=0.10$ and $\beta=0.95$. In the LVD experiment the value $Q=0.18$ and the corresponding yields $Y_{L S}=(3.3 \pm 0.5) \times 10^{-4}, Y_{F e}=(16.4 \pm 2.3) \times 10^{-4}$ were obtained under detection conditions $b$ ). Efficiencies $\eta_{L S}=0.75, \eta_{F e}=0.65$ were taken from [17]. Thus, the $Y_{L S}$ values from the reviewed papers exceed the corrected magnitudes by $\sim 30 \%$ (the table, Fig. 10).

The recent LVD results have been presented in 11]: $Y_{L S}=(3.2 \pm 0.2) \times 10^{-4}, Y_{F e}=(19 \pm 1) \times 10^{-4}$. The yield values were obtained based on the data of counters without triggering pulses to avoid some methodical effect reducing neutron detection efficiency in a counter

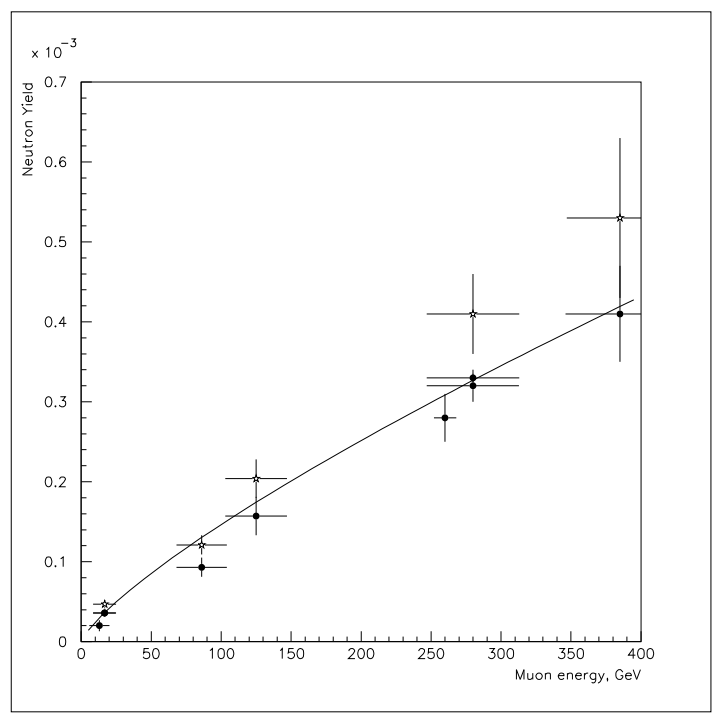

FIG. 1: Dependence of the neutron yield on muon energy for scintillator. The curve is a function $Y_{n}=4.03 \times 10^{-6} \bar{E}_{\mu}^{0.78}$ fitting the experimental points (filled circles), open stars are uncorected data.

with triggering pulse. In this case the efficiencies are $\eta_{L S}=0.0075, \eta_{F e}=0.0107$. All quantities $(Q, \eta, \bar{l})$ except for the starting number $N^{\text {det }}$ of the detected neutrons were calculated by the Monte Carlo method.

Formula for the muon-induced neutron yield.- The data collected in the table including early measurements [26], 27], 28], 29], 30] with iron and lead were analyzed using the conventional approach: $\alpha$ and $\beta$ are constants independent of $\bar{E}_{\mu}$ and $A$, correspondingly. Using independence of $\alpha$ on $\bar{E}_{\mu}$, for any A we can reduce the yield $Y\left(\bar{E}_{\mu}\right)$ values to a certain arbitrarily chosen energy $\bar{E}_{\mu}^{*}$ and calculate the average value of $\left\langle Y\left(\bar{E}_{\mu}\right)\right\rangle$ : for $\bar{E}_{\mu}^{*}=16.7 \mathrm{GeV}\left\langle Y_{L S}\right\rangle=0.34$ (averaged over nine values), $\left\langle Y_{F e}\right\rangle=1.70$ (averaged over seven values), $\left\langle Y_{P b}\right\rangle=6.33 \times 10^{-4} \mathrm{n} / \mu /\left(\mathrm{g} / \mathrm{cm}^{2}\right)$ (averaged over eight values). The ratio $\left\langle Y_{L S}\right\rangle /\left\langle Y_{F e}\right\rangle$ is consistent with $\beta=0.95$, while $\left\langle Y_{L S}\right\rangle /\left\langle Y_{P b}\right\rangle$ with $\beta=0.97$ and $\left\langle Y_{F e}\right\rangle /\left\langle Y_{P b}\right\rangle$ with $\beta=1.00$. The large $\beta$ values in the last two cases are mostly associated with excessive yield $Y_{P b}=116 \times 10^{-4}$ in experiment 30 .

The table data presented in Fig. 2 can be described by the expression

$$
Y_{n}\left(A, \bar{E}_{\mu}\right)=c A^{\beta} \bar{E}_{\mu}^{\alpha},
$$

where $\beta=0.95, \mathrm{c}$ is constant. Using the independence of $\beta$ on $\mathrm{A}$ and assuming $\beta=0.95$, the $Y_{F e}\left(\bar{E}_{\mu}\right)$ and $Y_{P b}\left(\bar{E}_{\mu}\right)$ data sets can be reduced to the $Y_{L S}\left(\bar{E}_{\mu}\right)$ set (Fig. 2, lower panel). Fitting the yield set of 24 values $Y_{L S}\left(\bar{E}_{\mu}\right)$ by expression $Y_{L S}=c(10.3)^{0.95} \bar{E}_{\mu}^{\alpha}$ we get the best agreement with the data at $c=(4.4 \pm 0.3) \times 10^{-7}$ 
and $\alpha=0.78 \pm 0.02$. The same values $c$ and $\alpha$, but at larger uncertainties, result from corrected LS data (Fig. 11. nine values).

The constant $\mathrm{c}$ is close to the value of the relative muon energy loss in nuclear interactions $b_{h}=4.0 \times 10^{-7}\left(\mathrm{~g} / \mathrm{cm}^{2}\right)^{-1}$. Therefore, $c$ is a relative muon energy loss for neutron production $c=b_{n}$ and it has the dimension $\left(\mathrm{g} / \mathrm{cm}^{2}\right)^{-1}$. Since neutrons are produced mainly in the em- and h- showers the constant $b_{n}$ should be associated with electromagnetic energy loss of muAgafonova2.texons $b_{\gamma}$ (em-shower generation mostly by means of bremsstrahlung) and nuclear loss $b_{h}$ (generation of h-showers where the main part of neutrons is produced). In the range from $\sim 100 \mathrm{GeV}$ to the extreme average muon energy underground $\sim 430$ $\mathrm{GeV}$ the generation of em- and h-showers is proportional to $\bar{E}_{\mu}$. The $b_{h}$ value does not depend on $\bar{E}_{\mu}$ and weakly depends on $\mathrm{A}: b_{h}=4.0 \times 10^{-7}$ for standard rock, $4.2 \times 10^{-7}$ for water [13]; the $b_{\gamma}$ loss varies slightly from $12.1 \times 10^{-7}$ up to $14.2 \times 10^{-7}$ for rock and from $8.2 \times 10^{-7}$ up to $9.9 \times 10^{-7}$ for water [13]. The $b_{\gamma}$ loss depend on the medium as $Z^{2} / A$. The proximity of the values $b_{n}$ and $b_{h}$ reflects, on one hand, the dominant role of h-showers in neutron production and, on the other, the practical constancy of $b_{n}$ in a wide range of $\bar{E}_{\mu}$ and $A$. The values of exponents $\alpha, \beta$ in equation (8) are determined by neutron production processes in showers: in emshowers $Y_{n} \propto \bar{E}_{\mu}^{1.0}$ [7], in h-showers $Y_{n} \propto \bar{E}_{\mu}^{0.75}$ [8], [10], [20]. Therefore, the resultant values $\alpha=0.78, \beta=0.95$ and $b_{n}=4.4 \times 10^{-7}$ obtained above are associated with the contributions of all neutron production processes, namely the shower generation by muons and the neutron production in showers via $\pi A, N A, \gamma A$-reactions.

Since the product $b_{n} E_{\mu}^{\alpha}\left[\mathrm{GeV} / \mu /\left(\mathrm{g} / \mathrm{cm}^{2}\right)\right]$ defines the muon energy loss for the neutron production, then, due to the yield dimension $\left[n / \mu /\left(g / \mathrm{cm}^{2}\right)\right]$, factor $A^{\beta}$ has the dimension of $[n / G e V]$.

The yield value is contained in the formula for the neutron production rate $r_{n}=I_{\mu}(H) \rho_{A} Y_{n}\left(E_{\mu}, A\right)\left(n / \mathrm{cm}^{3} c\right)$, where $I_{\mu}(H)\left(\mu / \mathrm{cm}^{2} c\right)$ is a muon intensity at a depth $H$ and $\rho_{A}$ is a medium density. Using this formula one can write the expression for rate $R_{n}$ of muon-induced neutrons in the detector and its shield consisting of different materials. The neutron rate for a material $A_{i}$ of a volume $v_{i}$ and mass $m_{i}$ is given by

$$
R_{n i}=v_{i} r_{n}=I_{\mu}(H) \rho_{A_{i}} v_{i} Y_{n_{i}}=I_{\mu}(H) m_{i} Y_{n_{i}}(n / c)
$$

For all materials of the detector and shield we have

$$
R_{n}=I_{\mu}(H) \Sigma m_{i} Y_{n_{i}}=I_{\mu}(H) b_{n} E_{\mu}^{\alpha} \Sigma m_{i} A_{i}^{\beta}(n / c)
$$

As it follows from equation (8) the neutron yield is highly dependent on $\bar{E}_{\mu}\left(\propto \bar{E}_{\mu}^{0.78}\right)$ and $A\left(\propto A^{0.95}\right)$. So, its value

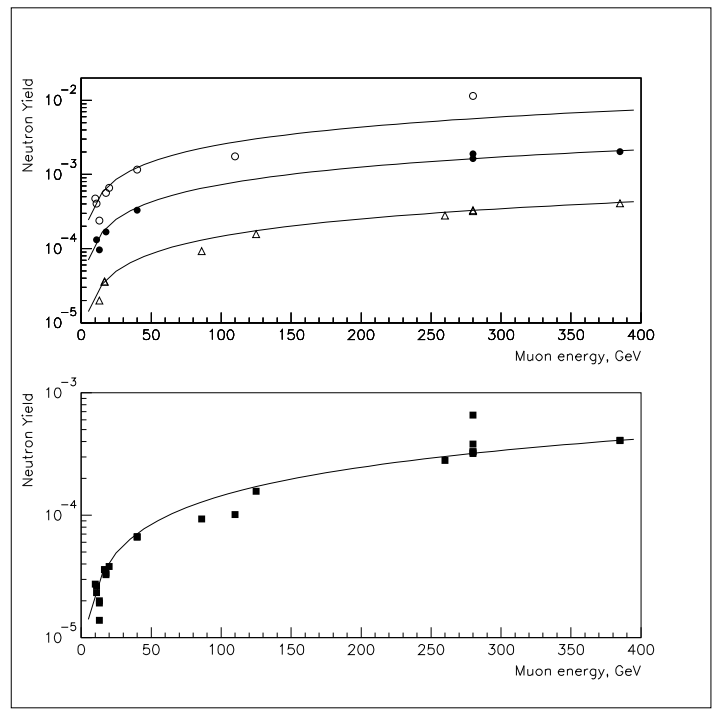

FIG. 2: Dependence of the neutron yield on $A$ and $\bar{E}_{\mu}$. Upper panel: experimental points for lead ( $A=207$, open circles), iron $(\mathrm{A}=56$, filled circles) and scintillator $(\mathrm{A}=10.3$, open triangles); the curves are functions $Y=c A^{\beta} \bar{E}_{\mu}$ at different $A$ and $c=4.4 \times 10^{-7}, \beta=0.95, \alpha=0.78$. Lower panel: neutron yield for scintillator; the experimental data for iron and lead are reduced to scintillator, the curve is a function $Y_{L S}=4.4 \times 10^{-7} 10.3^{0.95} \bar{E}_{\mu}^{0.78}$

for the heavy material ( $\mathrm{Fe}, \mathrm{Pb}$ ) can be used for experimental determination of $\bar{E}_{\mu}$ at any overburden topography and rock composition. The accuracy of the procedure might be not worse than in finding $\bar{E}_{\mu}$ by formulae in Ref. [13], 14], 15]. An approximation with constant parameters $b_{n}=4.4 \times 10^{-7} \mathrm{~cm}^{2} / \mathrm{g}, \alpha=0.78, \beta=0.95$ allows to use the formula (8) to calculate the yield for any $\bar{E}_{\mu}$ and $A$ in underground experiments. Since all nuclear effects produced by muons in the matter, including the production of radionuclides, are proportional to the neutron yield value the formula (8) is universal. However, the magnitudes of the parameters are determined by the contributions of nuclear and electromagnetic processes and therefore albeit weakly but depend on $\bar{E}_{\mu}$ and $A$. Due to the increasing requirements to the accuracy of the background determination in underground experiments the study of the neutron yield remains actual. This work was supported in part by the Russian Foundation for Basic Research grants 12-02-00213-a,12-0212127-ofi-m and SSh-871.2012.2.

* Electronic address: agafonova@lngs.infn.it

$\dagger$ Electronic address: malgin@lngs.infn.it

[1] Y.-F. Wang et al., Phys. Rev. D 64, 013012 (2001).

[2] H. M. Araujo et al., Nucl. Instrum. Methods A 545, 398 (2005).

[3] D. -M. Mei and A. Hime, Phys. Rev. D 73, 053004 (2006). 
[4] A. S. Malgin and O. G. Ryazhskaya, Phys. of Atomic Nuclei, 71, 10, 1769 (2008).

[5] O. G. Ryazhskaya and G. T. Zatsepin, Izv. AN USSR, Ser.Phys.,29,1946 (1965).

[6] L. B. Bezrukov et al., Sov. J. Nucl. Phys. 17, 51 (1973).

[7] R. I. Enikeev et al., Sov. J. Nucl. Phys.46, 1492 (1987).

[8] M. Aglietta et al., Nuovo Cimento C 12, 467 (1989).

[9] G. V. Gorshkov, V. A. Zyabkin, and R. M. Yakovlev, Sov. J. Nucl. Phys. 13, 450 (1971).

[10] C. Grupen, A. W. Wolfendale, and E. C. Young, Nuovo Cimento B 10, 144 (1972).

[11] R. Persiani, Ph. D. thesis (University of Bologna, 2011).

[12] S. Abe et al., Phys. Rev. C 81, 025807 (2010).

[13] V. I. Gurentsov, Preprint INR RAS, Moscow P-0380 (1984).

[14] P. Lipari and T. Stanev, Phys. Rev. D44, 3543 (1991).

[15] Donald E. Groom et al., At Data Nucl. Data Tables 78, 183 (2001).

[16] K. Zbiri, Nucl. Instrum. Methods A615, 220 (2010).

[17] N. Yu. Agafonova et al., (LVD Coll.) J. Phys.: Conf. Ser.409 012139 (2013).

[18] R. Hertenberger, M. Chen, and B. L. Dougherty, Phys.Rev. C 52, 3449 (1995).

[19] F. Boehm et al., Phys. Rev. D 62, 072002 (2000).
[20] O. G. Ryazhskaya, Diss doctor INR RAS, Moscow,(1986).

[21] N. Yu. Agafonova et al., (LVD Coll.) Izv. RAN, Ser. fiz., 69, 400 (2005)[Bull. Russ. Akad. Sci. Phys. 69, . (2005)].

[22] N. Yu. Agafonova et al., (LVD Coll.) Izv. RAN, Ser. fiz., 75, 437 (2011)[Bull. Russ. Akad. Sci. Phys. 75, 408 (2011)].

[23] M. Aglietta et al., in Proceedings of the XXVI ICRC, Salt Lake City, 244 (1999); hep-ex/9905047.

[24] V. A. Kudryvtsev et al., Nucl. Instrum. Methods A505, 688 (2003).

[25] A. S. Malgin, Preprint INR RAS 1333/2012, Moscow (2012).

[26] M. Annis, H. C. Wilkins, and J. D. Miller, Phys. Rev. 94, 1038 (1954).

[27] L. Bergamasco, Nuovo Cimento B 66, 120 (1970).

[28] G. V. Gorshkov and V. A. Zyabkin, Sov. J. Nucl. Phys. 7, 470 (1968).

[29] G. V. Gorshkov and V. A. Zyabkin, Sov. J. Nucl. Phys. 12, 187 (1970).

[30] L. Bergamasco, S. Costa, and P. Picchi, Nuovo Cimento A 13, 403 (1973). 